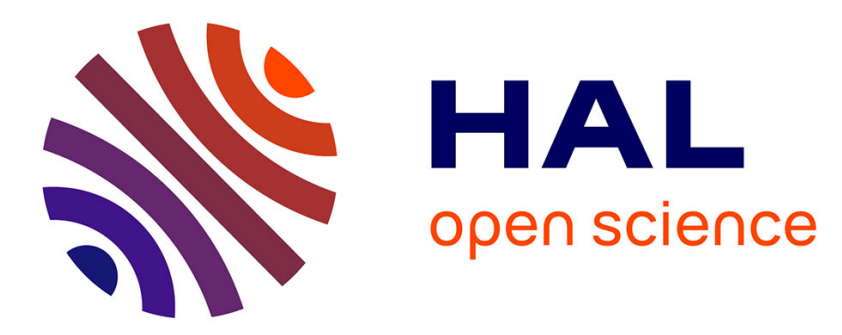

\title{
Optical nanoscopy with excited state saturation at liquid helium temperatures
}

\author{
Bin Yang, Jean-Baptiste Trebbia, Reenu Baby, Philippe Tamarat, Brahim \\ Lounis
}

\section{- To cite this version:}

Bin Yang, Jean-Baptiste Trebbia, Reenu Baby, Philippe Tamarat, Brahim Lounis. Optical nanoscopy with excited state saturation at liquid helium temperatures. Nature Photonics, 2015, 10.1038/nphoton.2015.152 . hal-01359111

\section{HAL Id: hal-01359111 \\ https://hal.science/hal-01359111}

Submitted on 1 Sep 2016

HAL is a multi-disciplinary open access archive for the deposit and dissemination of scientific research documents, whether they are published or not. The documents may come from teaching and research institutions in France or abroad, or from public or private research centers.
L'archive ouverte pluridisciplinaire HAL, est destinée au dépôt et à la diffusion de documents scientifiques de niveau recherche, publiés ou non, émanant des établissements d'enseignement et de recherche français ou étrangers, des laboratoires publics ou privés. 


\title{
Optical Nanoscopy with Excited State Saturation at Liquid Helium Temperatures
}

\author{
B. Yang ${ }^{1,2}$, J.-B. Trebbia ${ }^{1,2}$, R. Baby ${ }^{1,2}$, Ph. Tamarat ${ }^{1,2}$ and B. Lounis ${ }^{1,2 *}$. \\ ${ }^{1}$ Univ Bordeaux, LP2N, F-33405 Talence, France \\ ${ }^{2}$ Institut d'Optique \& CNRS, LP2N, F-33405 Talence, France \\ ”brahim.lounis@u-bordeaux.fr
}

Optical resolution of solid-state single quantum emitters at the nanometer scale is a challenging step towards the control of delocalized states formed by strongly and coherently interacting emitters. We developed a simple super-resolution optical microscopy method operating at cryogenic temperatures, which is based on optical saturation of the excited state of single fluorescent molecules with a doughnut-shaped beam. Sub-10 $\mathrm{nm}$ resolution is achieved with extremely low excitation intensities, a million times lower than those used in room temperature STED microscopy. Compared to super-localization approaches, our technique offers a unique opportunity to superresolve single molecules having overlapping optical resonance frequencies, paving the way to the study of coherent interactions between single emitters and to the manipulation of their degree of entanglement.

The controlled, coherent manipulation of quantum systems is an important challenge in modern science, with significant applications in quantum technologies ${ }^{1}$. Solid-state quantum emitters such as single molecules ${ }^{2}$, quantum $\operatorname{dots}^{3,4}$ and defect centers in diamond ${ }^{5}$ are promising candidates for the realization of quantum bits and quantum networks. Arrays of strongly interacting solid-state quantum systems are indeed appealing platforms for the preparation of collective delocalized states formed by the entanglement of individual emitters. Since coupling mechanisms such as dipole-dipole or tunneling occur on a nanometer scale, it is crucial to develop experimental schemes which optically resolve quantum emitters at this scale and allow the manipulation of their degree of entanglement ${ }^{6,7}$. The formation of collective quantum states from coupled optical emitters being a general phenomenon, these experimental schemes can also be useful for the study of many other systems including light harvesting complexes ${ }^{8,9}$, polymer conjugates ${ }^{10}$, quantum dots molecules ${ }^{11}$ and hybrid systems ${ }^{12}$.

Quantum emitters efficiently coupled to a photonic nanocavity with a small mode-volume ${ }^{13,14}$ or to a plasmonic structure ${ }^{15}$ also constitute promising systems for the realization of integrated solid-state 
devices for quantum photonics. To utilize such exceptional characteristics in scalable on-chip circuits ${ }^{16}$, efficient and deterministic coupling to photonic structures requires simple optical techniques for nanometer localization of the emitters with respect to the nanostructures.

In this context, organic fluorescent molecules embedded in well chosen solid matrices at liquid helium temperatures behave as simple two-level systems with a fluorescence quantum yield close to unity, thus offering optical properties of a test-bench system for quantum optics ${ }^{17-21}$. The absorption spectrum of a molecule presents a sharp zero-phonon line (ZPL), which is characterized by an extremely low saturation intensity (of the order of $1 \mathrm{~W} . \mathrm{cm}^{-2}$ ). In many well-studied host-guest systems, its linewidth reaches its fundamental lower bound determined by the emitting state inverse lifetime ${ }^{22}$. Moreover, the photostability of organic molecules is these systems is excellent and allows continuous optical measurements over days ${ }^{23}$.

Although the various super-resolution microscopy techniques ${ }^{24-29}$ are now commonly used at room temperature, e.g. in biological applications, their extension to low temperatures is in its infancy $^{30-32}$, mainly because of the inherent experimental complications of cryogenics and unsuitable photo-physical properties of usual markers at these temperatures. At liquid helium temperatures, two approaches have been developed to localize individual aromatic molecules with a lateral accuracy well below the diffraction limit. The first one is based on the spectral selection of single molecules with ultra-high-resolution laser spectroscopy and the analysis of the intensity distribution of their image spots on a CCD camera ${ }^{33}$. This method of localization of emission spot centers is simple but fails to super-resolve strongly coupled molecules, which generally have overlapping optical resonances. The second method is an experimental "tour de force" which combined fluorescence excitation spectroscopy and a scanning probe electrode inducing an inhomogeneous electric field used to record Stark-shift maps of single molecule lines ${ }^{6}$. In this work, we describe a cryogenic super-resolution optical microscopy method based on excited state saturation (ESSat) of individual fluorescent molecules. We show that ESSat microscopy is a simple technique, which can achieve sub-ten nanometer resolutions at low light intensities. It also provides a unique possibility to super-resolve single molecules having overlapping ZPLs. 
The host-guest system chosen in this experiment consists in dibenzanthanthrene (DBATT) molecules embedded in an octadecane Shpolskii matrix ${ }^{22}$, where single molecules exhibit narrow ZPL widths determined by the inverse lifetime of the excited state (Full-Width at Half- Maximum FWHM 20MHz) at liquid helium temperature, and weak saturation intensities $I_{S}$ of few W.cm ${ }^{-2}$ (see Figure 1a). The principle of the ESSat imaging technique is presented in Figure 1b. A single molecule is scanned through a focused doughnut-shaped laser beam (LG01 Laguerre-Gaussian mode) tuned to resonance while its fluorescence is recorded. For laser intensities much smaller than $I_{S}$, i.e. in the linear regime, the fluorescence signal is proportional to the light intensity and the image of the molecule reproduces the intensity distribution of the doughnut beam. When the laser intensity is increased, the saturation of the molecular transition leads to a broadening of the fluorescence image and to a sharpening of the dip at the doughnut beam center. If the resolution is defined as the FWHM of the central dip, it will be given by the diffraction limit in the linear excitation regime, while it will drop well below this limit with the onset of optical saturation. Approximating the intensity distribution of the doughnut-beam central region as $I(r)=I_{D} \sin ^{2}(N A \pi r / \lambda)$, the optical resolution is then given by:

$$
\Delta \mathrm{r}=\frac{2 \lambda}{\pi N A} \arcsin \frac{1}{\sqrt{2+I_{D} / I_{S}}}
$$

where $I_{D}$ is the intensity maximum of the doughnut beam and NA the numerical aperture of the microscope objective. At high saturations $\left(I_{D} \gg I_{S}\right)$, the resolution $\Delta \mathrm{r}$ decreases as $\sqrt{I_{S} / I_{D}}$.

The experimental setup of the ESSat microscope is depicted in Figure 2a. A home-built scanning confocal microscope is used to image single molecules excited at their ZPLs (around $\lambda \sim 589$ $\mathrm{nm}$ ) with a tunable single frequency dye laser. The microscope is based on a 0.95 NA objective, which is inserted in a helium cryostat together with the sample mounted on a piezo-scanner. The DBATTdoped octadecane film is spread over a glass coverslip with a thickness less than $10 \mu \mathrm{m}$. The emitted photons are filtered from the scattered excitation light by a band-pass filter (60 nm FWHM) and sent to a single-photon-counting avalanche photodiode. The doughnut-shaped excitation beam is obtained using a spatial light modulator (SLM), which imprints to the beam a vortex mask that includes an angular phase pattern varying azimuthally from 0 to $2 \pi$ with a point of undefined phase. The 
polarization of the doughnut beam is set circular using a quarter wave plate. To obtain a diffractionlimited doughnut spot with an azimuthally homogeneous intensity distribution around a zero-intensity center, we use the SLM to correct the optical aberrations of the objective, which are induced by mechanical strains at low temperatures (see Supplementary Information Figure S1). After corrections, the intensity distribution is nearly isotropic around the central dip, with azimuthal intensity variations lower than $20 \%$. Furthermore we could reduce the central intensity minimum of the doughnut beam to less than $10^{-4}$ of the maximum intensity (see below).

Fluorescence images of a single DBATT molecule excited with a Gaussian beam and with a doughnut beam at different laser intensities are displayed in Figure 3a. In the two upper images recorded in the linear regime, diffraction-limited spots mimicking the profiles of the excitation beams are obtained. The two lower images clearly show the expected sharpening of the central dip with increasing intensities of the doughnut beam. The experimental ESSat images are in excellent agreement with simulated images (see Supplementary Information Figure S2). From the crosssectional profiles of the images, we plot the evolution of the ESSat microscopy resolution as a function of the excitation intensity $I_{D}$ (see Figure $3 \mathrm{~b}$ and Supplementary Information Figure S3). The experimental data are well reproduced by equation (1) using the saturation intensity of the molecule $I_{S}=1.3 \mathrm{~W} . \mathrm{cm}^{-2}$ determined from saturation measurements as performed in Figure 1a. Clearly, sub 10 $\mathrm{nm}$ resolutions can be achieved.

Figure $3 \mathrm{c}$ and $3 \mathrm{~d}$ show the ESSat image and the cross-sectional profile of another molecule recorded at an intensity $I_{D}=13 \mathrm{~kW} . \mathrm{cm}^{-2}$, which is four orders of magnitude larger than $I_{S}$. In this case, we achieve an optical resolution of $4.4 \mathrm{~nm}$, close to the expected value of $3.8 \mathrm{~nm}$ determined by equation (1). This resolution is about a hundredth of the diffraction limit $(380 \mathrm{~nm})$ and approaches the size of the molecule $(\sim 1.5 \mathrm{~nm})$. It is noteworthy that these resolutions are lower than the best achieved at room temperature with color centers in diamond using $\mathrm{STED}^{34}$ and ground state depletion ${ }^{35}$ microscopies. Furthermore these resolutions are obtained with extremely low laser intensities (about ten $\mathrm{kW} / \mathrm{cm}^{2}$ ), more than five and more than three orders of magnitude weaker than those used at room temperature, respectively. Mechanical vibrations of the sample and objective holder in the cryostat set 
the current resolution limit of our ESSat microscope. Interestingly, we found that the fluorescence signal drops to about 0.4 of the maximal value in the central dip of the image. This reflects a residual intensity as low as 7. $10^{-5} I_{D}$, allowing super-resolution imaging of single molecules with a good contrast.

Direct ESSat microscopy gives "negative" images in the sense that the locations of the molecules are given by fluorescence minima. When more than one molecule are excited in the laser focal spot, their fluorescence signals overlap, which elevates the signal minima and therefore degrades the images contrast. To restore "positive" images, we overlap on the saturating doughnut beam a weak Gaussian beam having a temporally modulated intensity, and record the amplitude of the fluorescence signal modulation ${ }^{35}$. The principle of this method called modulated-ESSat microscopy is presented in Figure 4a. For a molecule located at the doughnut center, the laser intensity is much smaller than $I_{S}$ and the fluorescence signal varies linearly with the Gaussian beam intensity, leading to a maximal amplitude of the fluorescence modulation. Away from the doughnut center, the molecule is strongly saturated and the modulation of the fluorescence signal becomes negligible. A demodulation procedure to extract the amplitude of the modulated signal yields "positive" super-resolved images of single molecules.

Figure $2 \mathrm{~b}$ shows the experimental setup of the modulated-ESSat microscope. The modulation of the Gaussian beam intensity is performed at a frequency of $1 \mathrm{kHz}(500 \mu$ s ON/OFF periods) with two acousto-optic-modulators (AOMs). The two AOMs diffract the Gaussian beam in opposite orders and induce a total frequency shift of $\sim 1 \mathrm{MHz}$ with respect to the frequency of the doughnut beam. Thus, beating between the two beams focused in the sample plane vanish over the integration time of the detection. Moreover this frequency difference is much smaller than the molecular linewidth to ensure that both beams have nearly identical detuning from the molecular transition frequency.

Figure $4 \mathrm{~b}$ and $4 \mathrm{c}$ shows respectively the direct- and modulated-ESSat images of the same molecule excited at moderate doughnut beam intensity $I_{D} \sim 26 \mathrm{~W} \cdot \mathrm{cm}^{-2}$. Both images show the same sub-diffraction resolution of $\sim 80 \mathrm{~nm}$. The background observed in Figure $4 \mathrm{c}$ arises from the photonnoise of fluorescence emitted by the molecule when saturated by the doughnut beam. Figure $4 \mathrm{~d}$ 
presents a modulated-ESSat image of a single molecule for which we achieved the best resolution at higher saturation. In this case, the cross-section profile of the image gives a resolution of $\sim 9 \mathrm{~nm}$ (Figure 4e). This value is twice larger than that obtained with direct-ESSat due to longer integration times over which residual vibrations of the cryogenic microscopy setup are integrated.

Finally, in contrast with super-resolution microscopy methods based on single molecule localization $^{32,33}$, we demonstrate that modulated-ESSat provides a unique capability to super-resolve non-blinking molecules with spectrally stable overlapping optical resonance. Two such molecules, which are not resolved with classical confocal microscopy in Figure 4f, are clearly super-resolved in Figure $4 \mathrm{~g}$ when imaged with modulated-ESSsat microscopy.

In conclusion, we developed a simple super-resolution microscopy method operating at cryogenic temperature and based on excited state saturation of single emitters excited with a doughnut-shaped beam. We showed that sub-10 nm spatial resolutions (approaching the size of the molecule) can be achieved with low laser intensities (of the order of $10 \mathrm{~kW} / \mathrm{cm}^{2}$ ) compared to room temperature STED nanoscopy methods. Super-resolution nanoscopy of single molecules with overlapping transition resonances open up the study of strongly interacting quantum emitters in the view of applications in quantum networks. Further improvements will aim at achieving threedimensional super-resolution at low temperatures.

\section{Acknowledgement:}

We acknowledge financial support from Région Aquitaine, the French Ministry of Education and Research, the Institut universitaire de France and the Agence Nationale de la Recherche (France Bio Imaging, Grant N ANR-10-INSB-04-01). 


\section{REFERENCES}

1. Nielsen, M. A. \& Chuang, I. L. Quantum Computation and Quantum Information. (Cambridge University Press, 2010).

2. Moerner, W. E. \& Orrit, M. Illuminating single molecules in condensed matter. Science 283, 1670-1676 (1999).

3. Berezovsky, J., Mikkelsen, M. H., Stoltz, N. G., Coldren, L. A. \& Awschalom, D. D. Picosecond Coherent Optical Manipulation of a Single Electron Spin in a Quantum Dot. Science 320, 349 (2008).

4. Press, D., Ladd, T. D., Zhang, B. \& Yamamoto, Y. Complete quantum control of a single quantum dot spin using ultrafast optical pulses. Nature 456, 218-221 (2008).

5. Jelezko, F. \& Wrachtrup, J. Single defect centres in diamond: A review. phys. stat. sol. (a) 203, 3207-3225 (2006).

6. Hettich, C. et al. Nanometer resolution and coherent optical dipole coupling of two individual molecules. Science 298, 385-389 (2002).

7. Kasprzak, J., Patton, B., Savona, V. \& Langbein, W. Coherent coupling between distant excitonsrevealed by two-dimensional nonlinearhyperspectral imaging. nature photonics $\mathbf{5}$, 57-63 (2010).

8. Herek, J. L., Wohlleben, W., Cogdell, R. J., Zeidler, D. \& Motzkus, M. Quantum control of energy flow in light harvesting. Nature 417, 533-535 (2002).

9. Engel, G. S. et al. Evidence for wavelike energy transfer through quantum coherence in photosynthetic systems. Nature 446, 782-786 (2007).

10. Hwang, I. \& Scholes, G. D. Electronic Energy Transfer and Quantum-Coherence in $\pi-$ Conjugated Polymers †. Chem. Mater. 23, 610-620 (2011).

11. Wu, J. \& Wang, Z. M. Quantum Dot Molecules. (Springer Science \& Business Media, 2013).

12. Xiang, Z.-L., Ashhab, S., You, J. Q. \& Nori, F. Hybrid quantum circuits: Superconducting circuits interacting with other quantum systems. Rev. Mod. Phys. 85, 623-653 (2013).

13. Badolato, A. et al. Deterministic coupling of single quantum dots to single nanocavity modes. Science 308, 1158-1161 (2005).

14. Arcari, M. et al. Near-Unity Coupling Efficiency of a Quantum Emitter to a Photonic Crystal Waveguide. Phys. Rev. Lett. 113, 093603 (2014).

15. Chang, D. E., Sørensen, A. S., Demler, E. A. \& Lukin, M. D. A single-photon transistor using nanoscale surface plasmons. Nature Physics 3, 807-812 (2007).

16. Northup, T. E. \& Blatt, R. Quantum information transfer using photons. nature photonics 8 , 356-363 (2014).

17. Tamarat, P. et al. Pump-probe experiments with a single molecule: ac-stark effect and nonlinear optical response. Phys. Rev. Lett. 75, 1514 (1995).

18. Brunel, C., Lounis, B., Tamarat, P. \& Orrit, M. Triggered source of single photons based on controlled single molecule fluorescence. Phys. Rev. Lett. 83, 2722 (1999).

19. Wrigge, G., Gerhardt, I., Hwang, J., Zumofen, G. \& Sandoghdar, V. Efficient coupling of photons to a single molecule and the observation of its resonance fluorescence. Nature Physics 4, 60-66 (2007).

20. Hwang, J. et al. A single-molecule optical transistor. Nature 460, 76-80 (2009).

21. Trebbia, J.-B., Tamarat, P. \& Lounis, B. Indistinguishable near-infrared single photons from an individual organic molecule. Phys. Rev. A 82, 063803 (2010).

22. Tamarat, P., Maali, A., Lounis, B. \& Orrit, M. Ten Years of Single-Molecule Spectroscopy. J. Phys. Chem. A 104, 1-16 (2000).

23. Basché, T., Moerner, W. E., Orrit, M. \& Wild, U. P. Single Molecule Optical Detection, Imaging. VCH, Weiheim (1996).

24. Hell, S. W. Far-field optical nanoscopy. Science 316, 1153-1158 (2007).

25. Betzig, E. et al. Imaging Intracellular Fluorescent Proteins at Nanometer Resolution. Science 313, 1642-1645 (2006). 
26. Rego, E. H. et al. Nonlinear structured-illumination microscopy with a photoswitchable protein reveals cellular structures at 50-nm resolution. Proceedings of the National Academy of Sciences 109, E135-E143 (2012).

27. Huang, B., Bates, M. \& Zhuang, X. Super-Resolution Fluorescence Microscopy. Annu. Rev. Biochem. 78, 993-1016 (2009).

28. Fujita, K., Kobayashi, M., Kawano, S., Yamanaka, M. \& Kawata, S. High-Resolution Confocal Microscopy by Saturated Excitation of Fluorescence. Phys. Rev. Lett. 99, 228105 (2007).

29. Godin, A. G., Lounis, B. \& Cognet, L. Biophysical Review. Biophysical Journal 107, 1777-1784 (2014).

30. Chang, Y.-W. et al. Correlated cryogenic photoactivated localization microscopy and cryoelectron tomography. Nat Meth 11, 737-739 (2014).

31. Kaufmann, R. et al. Super-resolution microscopy using standard fluorescent proteins in intact cells under cryo-conditions. Nano Lett. 14, 4171-4175 (2014).

32. Weisenburger, S. et al. Cryogenic colocalization microscopy for nanometer-distance measurements. ChemPhysChem 15, 763-770 (2014).

33. van Oijen, A. M., Köhler, J., Schmidt, J., Müller, M. \& Brakenhoff, G. J. 3-Dimensional superresolution by spectrally selective imaging. Chemical Physics Letters 292, 183-187 (1998).

34. Rittweger, E., Han, K. Y., Irvine, S. E., Eggeling, C. \& Hell, S. W. STED microscopy reveals crystal colour centres with nanometric resolution. nature photonics 3, 144-147 (2009).

35. Rittweger, E., Wildanger, D. \& Hell, S. W. Far-field fluorescence nanoscopy of diamond color centers by ground state depletion. EPL 86, 14001 (2009). 

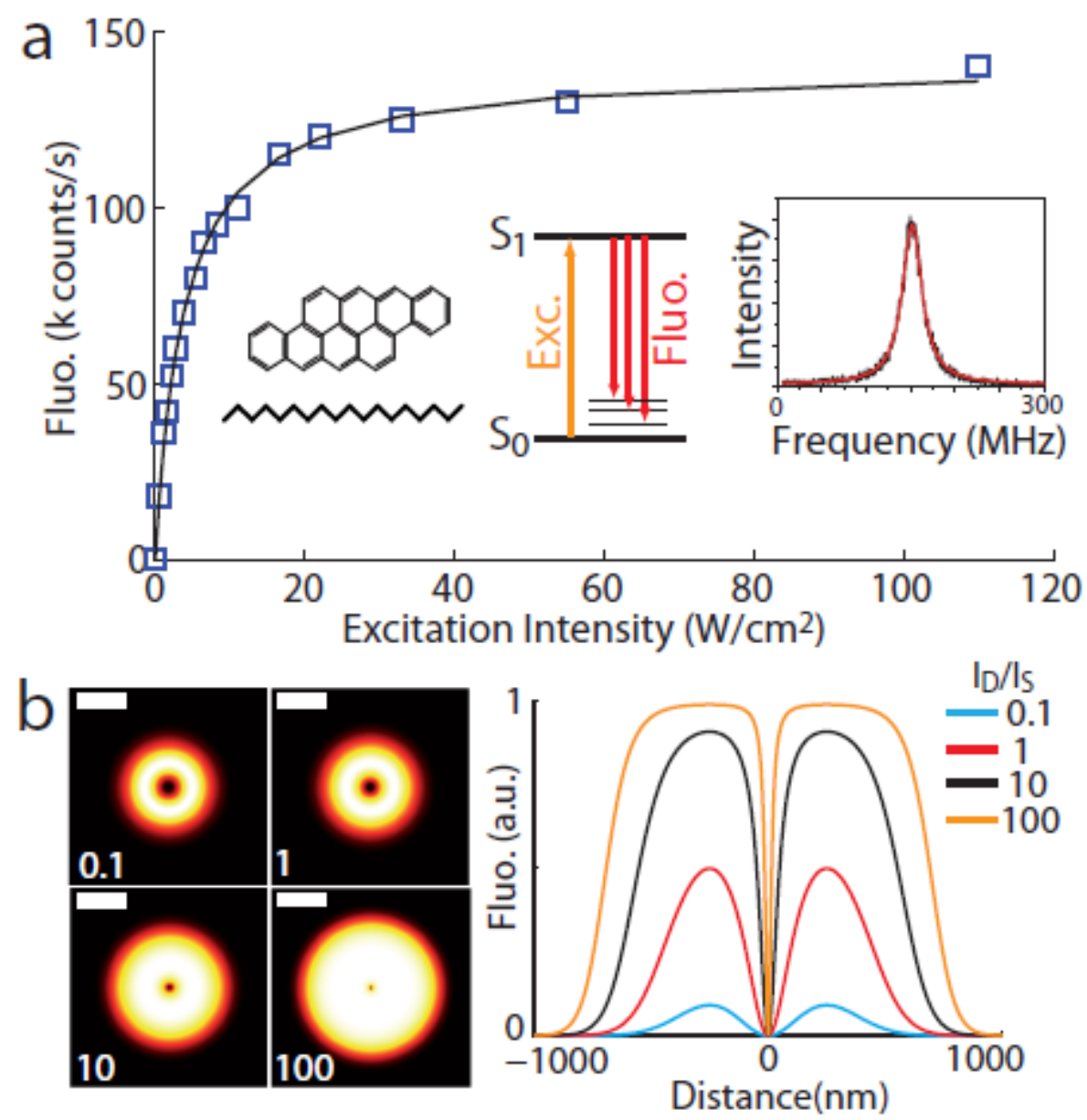

Figure 1: Principle of ESSat microscopy. a, Saturation curve obtained for a single DBATT molecule excited at resonance at the zero-phonon line (ZPL), recorded at $\mathrm{T}=2 \mathrm{~K}$. Fitting the saturation curve at resonance with $S=S_{\infty}\left(I / I_{S}\right) /\left(1+I / I_{S}\right)$, we extract a saturation intensity $I_{S}=3.8 \mathrm{~W} \cdot \mathrm{cm}^{-2}$. Insets from left to right: Chemical structures of DBATT and octadecane. Energy levels of the DBATT molecule, excited on the singlet-singlet purely electronic transition $\mathrm{S}_{0}-\mathrm{S}_{1}$. Fluorescence photons are emitted on transitions from $\mathrm{S}_{1}$ to vibrational levels of the ground electronic state $\mathrm{S}_{0}$. Fluorescence excitation spectrum of a single DBATT molecule in the low excitation regime, showing a Lorentzianshaped ZPL with FWHM $\sim 20$ MHz. b, Fluorescence images and corresponding cross-section profiles calculated for a molecule excited at different doughnut beam intensities. Scale bars: $600 \mathrm{~nm}$. 


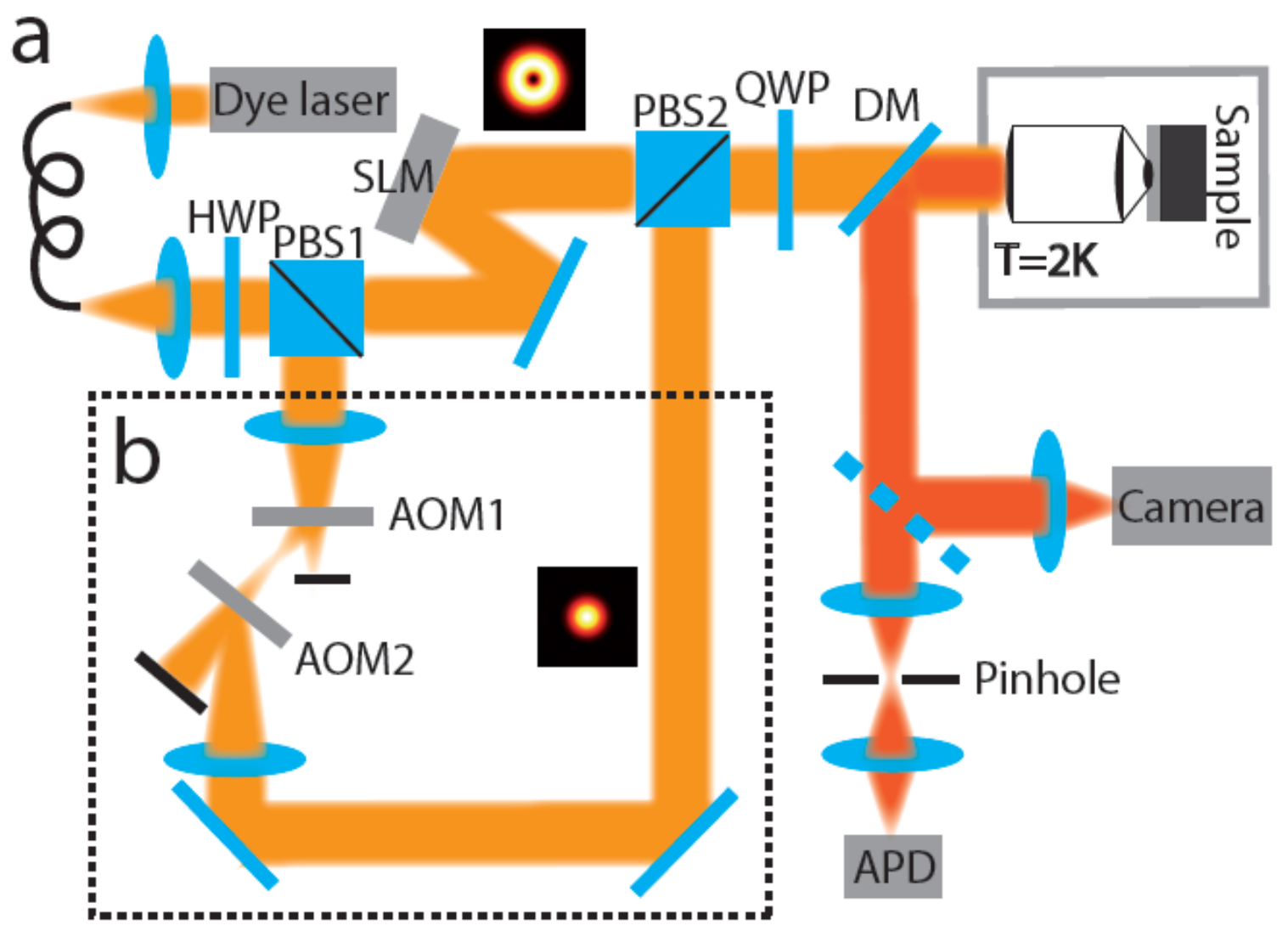

Figure 2: Experimental setup of the cryogenic ESSat microscope.

a, Direct ESSat microscopy setup. A high-NA objective and a sample mounted on a piezo-scanner are inserted in a helium cryostat cooled at $2 \mathrm{~K}$. DBATT molecules are excited with a tunable singlefrequency dye laser at $589 \mathrm{~nm}$. The doughnut-shaped beam is generated with a spatial light modulator. A CDD camera is used to characterize the spatial profile of the doughnut beam. A single-photoncounting avalanche photodiode (APD) records the fluorescence emitted by molecules. $\mathbf{b}$, ModulatedESSat microscopy module. The excitation beam is split by a polarizing beamsplitter (PBS1). The reflected beam is temporally modulated in intensity by two acoustic-optic modulators (AOM), and then recombined with the doughnut-shaped beam with PBS2. Notations: HWP: half-wave plate; QWP: quarter-wave plate; DM: dichroic mirror. 

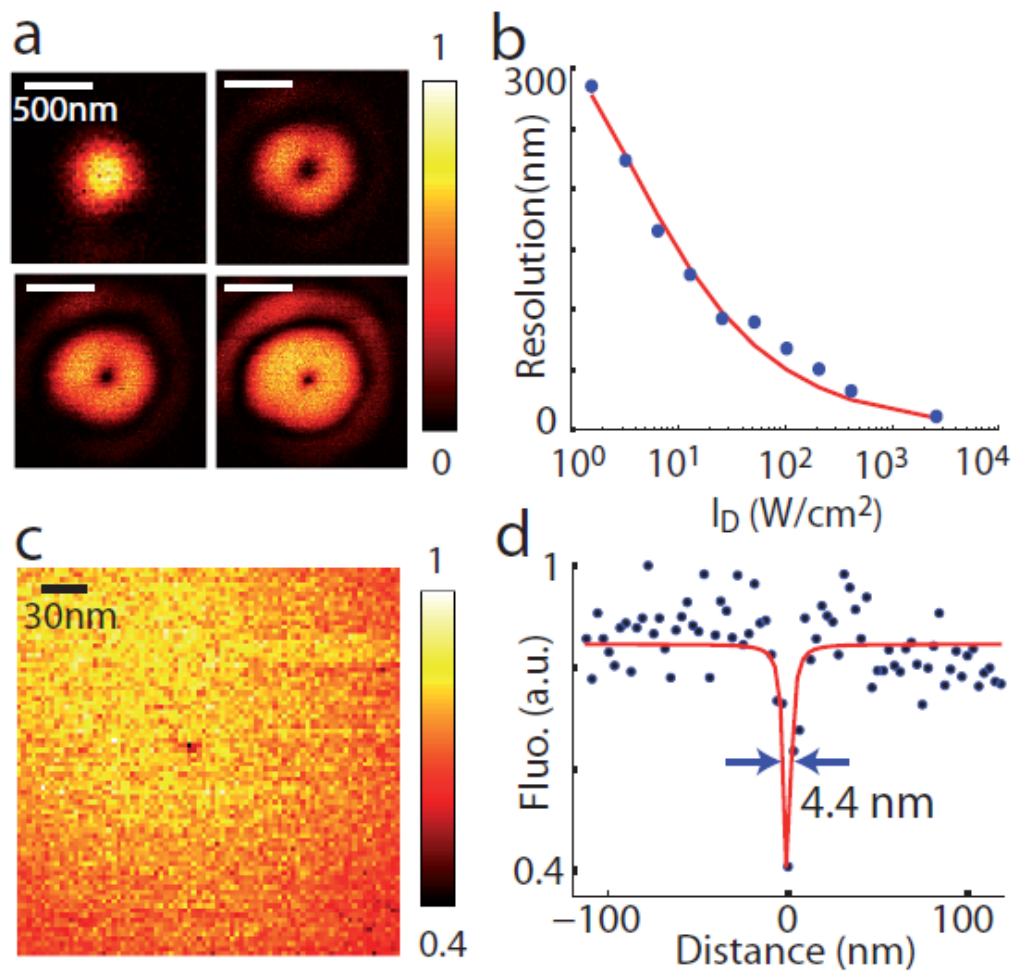

\section{Figure 3 Experimental results of direct ESSat microscopy.}

a, Images of a single DBATT molecule obtained with a Gaussian beam (upper left image) and with the doughnut beam at different intensities $\left(I_{D}=0.8,3.3\right.$ and $\left.13 \mathrm{~W} . \mathrm{cm}^{-2}\right)$. b, Direct-ESSat resolution as a function of the doughnut intensity. Blue spots: experimental data; red line: theoretical fit (see text). c, Direct-ESSat image of another molecule recorded with $I_{D}=13 \mathrm{~kW} . \mathrm{cm}^{-2}$. Pixel dwell time: $10 \mathrm{~ms}$. d, Cross-sectional profile of the image (c), showing a resolution of $4.4 \mathrm{~nm}$. 

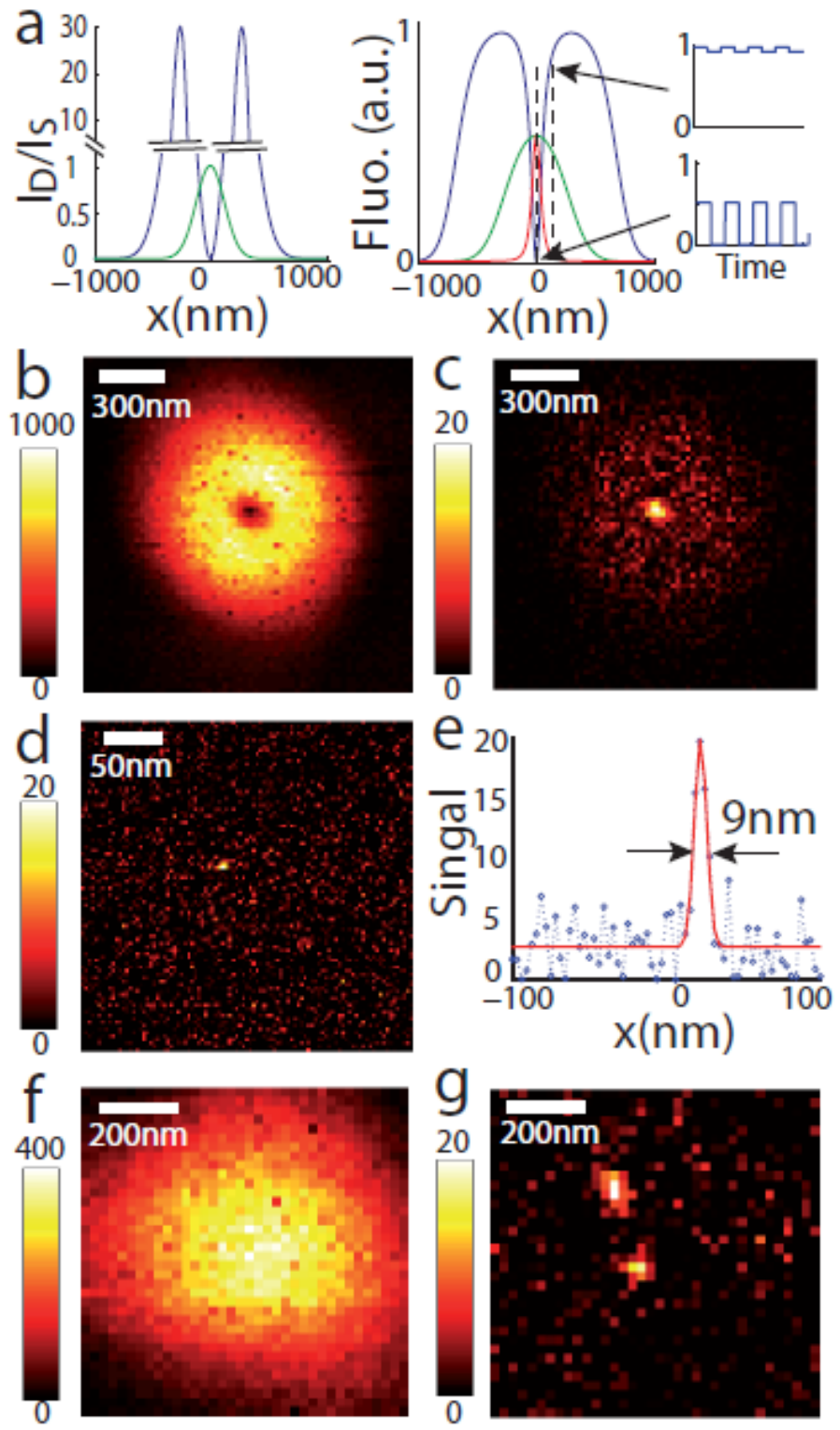

Figure 4 Principle and experimental results of modulated-ESSat microscopy.

a, left: Intensity profiles of the Gaussian beam (green curve) and the doughnut beam (blue curve); right: Calculated fluorescence signal profiles of a molecule excited with the doughnut beam (blue curve) and with the Gaussian beam (green curve). The temporal modulation of the fluorescence is shown for two different positions of the molecule. The amplitude of the fluorescence modulation is plotted as a function of the distance between the molecule and the doughnut center (red curve). b,c, Direct- and modulated-ESS images of a molecule for $I_{D}=26 \mathrm{~W} . \mathrm{cm}^{-2}$ and $I_{G}=10 \mathrm{~W} . \mathrm{cm}^{-2}$. d, Modulated-ESSat image of another molecule recorded at $I_{G}=170 \mathrm{~W} \cdot \mathrm{cm}^{-2}$ and $I_{D}=2.2 \mathrm{~kW} \cdot \mathrm{cm}^{-2}$. e, Cross-section profile of image (d), with a FWHM of $9 \mathrm{~nm}$. f, g, Confocal and modulated-ESSat images of two molecules with overlapping resonances, recorded at $I_{G}=25 \mathrm{~W} . \mathrm{cm}^{-2}$ and $I_{D}=260 \mathrm{~W} . \mathrm{cm}^{-2}$. Pixel dwell time: $3 \mathrm{~ms}(\mathbf{b}, \mathbf{f})$ and $25 \mathrm{~ms}(\mathbf{c}, \mathbf{d}, \mathbf{g})$. 